\title{
To Use or Not to Use Artificial Intelligence? A Framework for the Ideation and Evaluation of Problems to Be Solved with Artificial Intelligence
}

\author{
Timo Sturm \\ Technical University of Darmstadt \\ sturm@is.tu-darmstadt.de
}

\author{
Mariska Fecho \\ Technical University of Darmstadt \\ fecho@is.tu-darmstadt.de
}

\author{
Peter Buxmann \\ Technical University of Darmstadt \\ buxmann@is.tu-darmstadt.de
}

\begin{abstract}
The recent advent of artificial intelligence (AI) solutions that surpass humans' problem-solving capabilities has uncovered AIs' great potential to act as new type of problem solvers. Despite decades of analysis, research on organizational problem solving has commonly assumed that the problem solver is essentially human. Yet, it remains unclear how existing knowledge on human problem solving translates to a context with problem-solving machines. To take a first step to better understand this novel context, we conducted a qualitative study with 24 experts to explore the process of problem finding that forms the essential first step in problem-solving activities and aims at uncovering reasonable problems to be solved. With our study, we synthesize emerged procedural artifacts and key factors to propose a framework for problem finding in AI solver contexts. Our findings enable future research on human-machine problem solving and offer practitioners helpful guidance on identifying and managing reasonable AI initiatives.
\end{abstract}

\section{Introduction}

In recent years, advances in artificial intelligence (AI) allowed machines to master problems previously dominated by humans: AIs defeated world's best human GO player [51], recognized images better than the average human [19], and beat some of the greatest human StarCraft II players [59]. Due to success stories like these, more and more organizations aim to explore how to use AIs' disruptive potential to improve their organizational performance [e.g., 5, 18, 49].

The technology that underlies such modern AI information systems (IS) is machine learning (ML) [9, 22]. Such ML-based AIs use ML algorithms to derive patterns from data and apply these patterns to new data to perform actions [9, 32, 43]. ML thus constitutes a new programming paradigm: With $M L$, algorithms derive solutions from data, instead of having humans manually solving problems and translating their solutions into code anymore [46]. The resulting handover of problem-solving activities to data-driven algorithms therefore requires us to reassess the role of IS in organizations and our knowledge on how to manage IS successfully [e.g., 40, 44].

One process that essentially drives and ensures an organization's progress and is thus crucial for its longterm survival is the act of problem solving [24, 29]; that is, the act of finding, solving, and implementing solutions for problems [4, 28]. For decades, scholars from various disciplines have analyzed problem solving from different perspectives [e.g., 12, 27]. Yet, such organizational studies have commonly assumed that the solver of organizations' problems is only human. With ML-based AI essentially representing a technology for machine-driven problem solving that organizations increasingly adopt $[9,32,43]$, this core assumption must be fundamentally questioned.

To take a first step to better understand how organizations can manage problem solving in the AI age, we explore how problem finding, which precedes the core problem solving activity and aims to identify relevant problems, translates to contexts where AIs act as problem solvers. To achieve this, we conducted a qualitative study with 24 experts that frequently conduct AI initiatives. We thus aim to answer:

(1) How can organizations find problems that are likely suited to be solved by ML-based AIs, and

(2) which central factors likely determine $M L$ based AIs' suitability for solving a problem?

\section{Theoretical Background}

Below, we first present key concepts and related work of problem solving and AI. We then integrate both research streams to form our study's objective.

\subsection{The Process of Problem Solving}

Problem solving, the act of uncovering problems and searching for effective solutions $[20,53,54,56]$, is 
considered a key activity in organizations [27, 29]. This process involves generating new, suitable ideas (i.e., both problems and solutions) at its core $[4,33$, 41], playing an essential role for organizations [7] as ideas stimulate organizational change [15].

One model that represents a solid synthesis of the widespread consensus of general stages of problemsolving processes [e.g., 27, 33, 41, 54] is the one originally proposed by Basadur et al. (1982) [4] based on Leavitt's (1975) [28] suggested tripartite model. Figure 1 illustrates this process that we describe below.

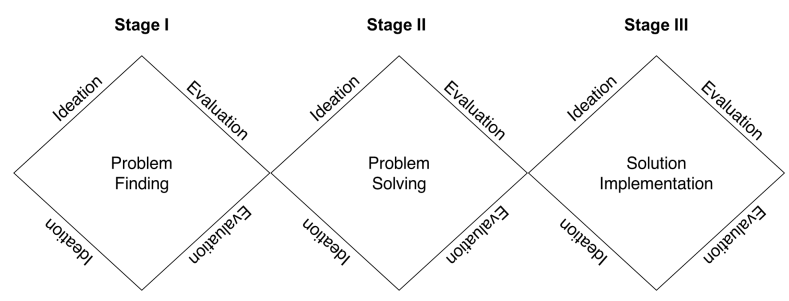

Figure 1. Problem-Solving Process [4]

The problem-solving process comprises three steps: First, the initial problem finding aims to recognize, identify, and construct problems. Then, the problem solving includes the search for suitable solutions by exploring potentially fitting solutions for given problems [34]. Finally, the solution implementation pursues to integrate selected solutions into organizational processes [35]. Following Osborn's (1953) [37] widely adopted divergence-convergence dualism (i.e., uncover choices and screen choices), each of the three above-mentioned stages also comprises a two-step subprocess [4, 37]: First, ideation aims to explore ideas. Depending on the three stages in Figure 1, this respectively refers to uncovering and constructing potential problems, solutions, and solution implementations. The subsequent evaluation then assesses the respective ideas that yielded from the ideation to distinguish good ideas from bad ones.

This process helps us to better understand the essential role of problem finding. Within this step, humans identify and construct problems on the basis of domain knowledge and prior experiences [10] to uncover problems together with related goals, possible problem-solving approaches, and restrictions [41]. Since ill-defined problems can contain characteristics that lead to unexpected or unsatisfactory results (e.g., selecting unsuitable approaches or missing relevant aspects), problem finding essentially affects the success of problem-solving activities [20,52].

For decades, scholars have examined how human problem solving, and in particular problem finding, translates to different contexts, such as the individual or group-level [e.g., 12, 27]. In recent years, research has also started to examine problem finding for creating solutions with digital technologies. As a result, several frameworks were proposed that focus on digital technologies in general [e.g., 6, 8, 23]. For instance, most closely related to our study, Vanauer et al. (2015) [58] propose an ideation framework for Big Data solutions. They found multiple procedural artifacts that comprise two ideation alternatives and several suitability assessments. Although ML-based AI also represents a digital technology, the existing frameworks neglect particularities that result from the unique problem-solving capabilities of ML-based AI.

\subsection{Artificial Intelligence \& Machine Learning}

A widely used conceptualization of $\mathrm{AI}$ is the intelligent agent; that is, anything that can perceive context information and autonomously act upon that through actuators [43]. The technique that organizations increasingly use to implement such agents' behavior is ML [9]: Intelligent agents based on ML-by us referred to as $M L$-based $A I$ - are based on algorithms that can identify patterns in data and use these patterns to act on new data [32]. Without ML, humans solve problems manually and codify their solutions into traditional non-ML IS. In contrast, MLbased AIs derive their own solutions for defined problems exclusively from data, rendering manual programming unnecessary $[9,46]$. While artificial general intelligence remains beyond reach, organizations increasingly use ML-based AI to solve narrow problems [9], sometimes achieving solutions that even surpass human problem-solving capabilities [e.g., 19, 51, 59]. Especially in contexts where tasks comprise a limited execution clarity, ML-based AI offers a great potential to explore available alternatives and evaluate their properties more extensively and precisely than their human counterparts that are more limited in their information processing capabilities [9].

To exploit this potential, organizations must understand how they can use ML-based AI to solve problems within their organizational contexts. Yet, existent research has only partially unveiled how organizations can use ML-based AI for their problemsolving activities. Thus far, scholars have intensively focused on understanding AI-driven problem solving to develop solutions for given problems: Professionals select and prepare data and also select and parametrize ML algorithms to frame a given problem and restrict potential solution designs. Next, they let the algorithms derive possible ML-based AI solutions and then evaluate the solutions. The professionals iteratively perform this process to eventually identify the best derivable AI solution $[9,14,32]$. Existing research has proposed multiple frameworks to capture this process from different perspectives [e.g., 3, 14, 26]. 
Although this research generally expects problems to exist that must be solved, only a few studies stand out that explore how organizations can actually uncover problems to be solved with ML-based AI. The most applicable study is the one of Brynjolfsson and Mitchell (2017) [9], in which they name basic criteria to identify suitable tasks for applying ML-based AI, but do not provide procedural guidance and only consider a single ML type (i.e., supervised ML). Besides, existing studies either regard this topic from a more strategic perspective to provide factors for MLbased AI adoption [e.g., 25, 39, 55, 57], or focus on separate AI particularities, such as research on fair [e.g., 1, 30, 42] and transparent AI [e.g., 13, 31, 38].

\subsection{The Need to Revisit Problem Finding}

The problem-solving process by Basadur et al. (1982) [4] offers a solid basis for exploring problemsolving activities in different contexts. Despite decades of research, humans have generally been considered the only actor that performs the second step within this process; that is, deriving appropriate solutions. As we are interested in understanding how the initial problem finding step translates to contexts where solutions are created by ML-based AI, we must reassess the fit of existent research with this novel context.

Moreover, research on the use of ML-based AI in organizational contexts has widely neglected the process of problem finding. Although AI research has conceptualized the development of ML-based AI solutions, the act of finding suitable problems has been widely overlooked so far. While some exceptions exist, corresponding studies do not provide procedural artifacts and mostly provide factors for more abstract or specific areas. Lastly, while problem-finding frameworks for digital technologies exist, they neglect to include relevant ML-based AI's particularities due to their divergent, more general technological focus.

So far, we miss insights to sufficiently explain how organizations can perform the initial, operative act of finding problems to be solved with ML-based AI. We therefore decided to conduct an explorative study to gather first evidence and propose a basic framework for problem finding within this novel context.

\section{Qualitative Research Methodology}

To explore central factors that influence the suitability of organizational problems for being suited to be solved with ML-based AI, we applied a qualitative research approach. In particular, we interviewed experts from the operational and management levels of different organizations that are highly involved in AI initiatives [17]. We then followed the steps of a directed content analysis [21] to contextualize problem finding for ML-based AI. According to Weber (1990) [60], content analysis can be used to categorize and evaluate qualitative data.

Based on the proposed principles by Sarker et al. (2013) [47], we formulated a semi-structed interview guideline that we used to lead the interviews. A high degree of coherence was ensured by discussing our definition of ML-based AI and selected use cases with each expert before every interview. We used semistructured questions as they ensure that all relevant questions are posed, while allowing the experts to freely share own experiences and opinions [36]. To examine various factors and procedural artifacts, our interview questions followed both an organizational and technological perspective to examine the essential ideation-evaluation process underlying problem finding [4]. Finally, our interview guide covered the following five sections: general information about the experts, ML-based AI particularities, organizational and technical requirements, identification and evaluation of AI usage scenarios, and potential benefits and risks related to the adoption of $\mathrm{AI}$ in organizational processes. The iterative approach during the interviews allowed a continuous adaptation of the initially defined questions. Thus, on the one hand, the focus of the investigation could be sharpened while, on the other hand, individual perceptions could be considered [36].

We selected interview partners, who have detailed experience in solving organizational problems with ML-based AI. We conducted 23 interviews with 24 experts from Europe and North America, including nine experts from user firms (i.e., that mainly purchase AI products) and 15 experts from provider firms to comply with data triangulation rules [17]. One interview included two experts. The experts cover developers, data scientists, managers, pre-sales consultants, and technical consultants, who regularly deal with the design and integration of prototypical or productive systems in different organizational contexts. We noticed that we reached theoretical saturation [17] during the last five interviews as they yielded no further insights and thus stopped interviewing.

The interviews were conducted either by telephone or face-to-face between December 2018 and April 2019. On average, each interview lasted 56 minutes. They were recorded, transcribed, and analyzed using the NVivo 12 software. In line with Saldaña (2009) [45], we performed an iterative multi-cycle coding process consisting of two coding cycles. The first cycle covered three types of coding: First, we used (1) attribute coding to extract essential information about participants and organizations. Then, we employed (2) hypothesis coding to determine and structure potential factors along insights of human problem finding. This 
step allowed us to stimulate code derivation by objectives and approaches of human problem finding that might be generalizable or ill-suited to the AI context. Finally, we applied (3) descriptive coding to identify new procedural artifacts and key factors that might extend the initial problem-finding process, allowing us to uncover AI-related particularities more independently from human problem finding. Since the first coding cycle resulted in a large number of factors (i.e., 11 procedural artifacts and 37 key factors), we used pattern coding in a second cycle to cluster similar constructs to form mutually exclusive and collectively exhaustive procedural artifacts and key factors.

This two-cycle coding process was performed individually and independently by two of the authors and two student assistants. All results were compared and integrated in discussions with all four parties until a consensus was reached: an initial framework emerged in which procedural artifacts were integrated into phases of problem finding and each procedural artifact was fortified with associated key factors. To achieve research rigor, the coding process and initial framework were validated in subsequent discussions between five IS researchers and three student assistants [17]. Additional data sources regarding problem finding for digital technology contexts and the use of AI in organizations [i.e., 2, 6, 8, 9, 16, 23, 48, 57, 58] were also considered to compare results with existing knowledge. Based on this data and investigator triangulation [17], the final framework was formed.

\section{Results}

With our study, we explored how problem finding translates to a context, in which the problem is aimed to be solved with ML-based AI. Our results show first evidence for fundamental procedural artifacts and related key factors. In our interviews, it got apparent that the fundamental phases of problem finding in a human solver context (i.e., ideation and evaluation) remain valid for an ML-based AI context. Moreover, we found that finding a problem for being solved with ML-based AI is determined by three central aspects; that is, a clear organizational purpose, available data, and technical particularities of ML-based AI. A model for problem finding in an ML-based AI context should therefore essentially follow an ideation and evaluation phase, while considering subphases driven by factors of the three central aspects.

Figure 2 illustrates the framework that emerged from our interviews. Its main structure follows a twophase character: An ideation phase first aims to uncover potential problems. Within this phase, problems are explored aiming to fulfill both a clear organizational purpose and availability of required data. The subsequent two-step evaluation phase then aims to assess the suitability of envisioned problems. The phase starts with evaluating problem substance of uncovered problems; that is, to generally be suited for ML-based AI. If this essential suitability can be confirmed, evaluating problem particularities follows to clarify whether and to which extent special features of ML-based AI solutions fit given problems.

Note that this evaluation phase focuses on narrowing down problems based on their suitability for being solved with ML-based AI from a technical point of view, which our experts deemed central to problem evaluation in the AI context. As with human problem finding, construct and content-wise organizational evaluations likely extend our conceptualized evaluation phase to further assess problems' adequacy for being solved with respect to an organization's objectives. As this is out of our study's scope, we leave it to future research to analyze potential aspects for such problem evaluation foci while we abstract these hereinafter.

Below, we present the derived concepts. As each concept is grounded on some degree of consensus between the experts, we also indicate this degree as percentage of experts focusing each respective concept.

\subsection{Ideation Phase}

Within our interviews, it got apparent that a worthwhile ML-based AI solution unites a reasonable organizational purpose, available required data, and the fulfillment of ML-based AIs' technical particularities. Otherwise, even if the defined problem is basically suited to be solved with ML-based AI, an implemented solution may end up not being used if no clear organizational purpose is included or the

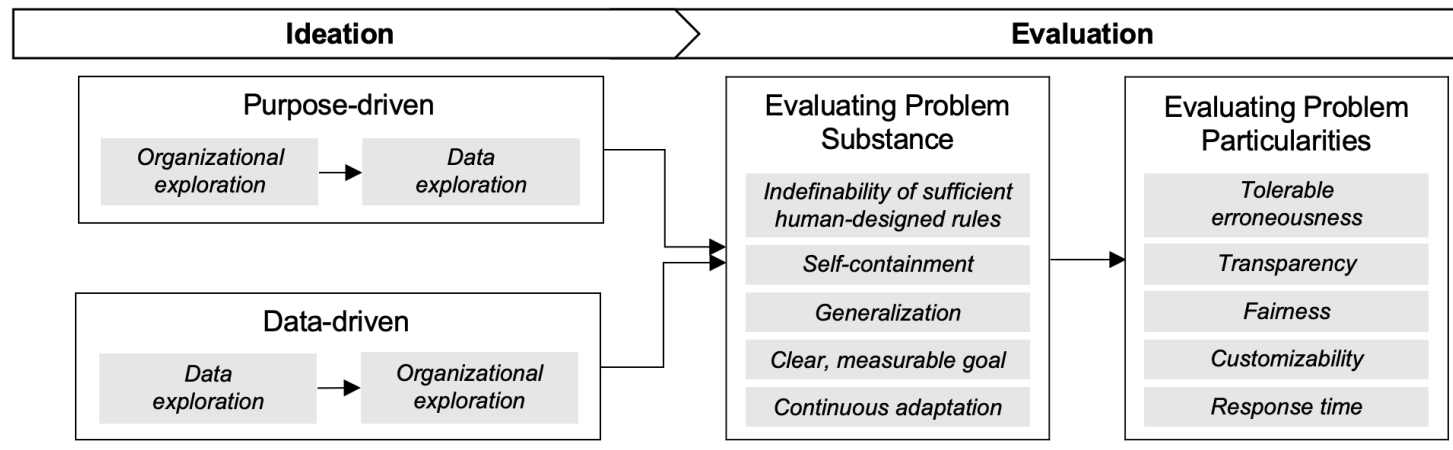

Figure 2. A framework for the ideation and evaluation of problems to be solved with ML-based AI 
implementation may fail if the required data turns out to be insufficient to create a functioning solution.

To increase the likelihood of finding suitable problems already in an early stage, the ideation phase should involve three essential elements: organizational exploration, data exploration, and an AI-specific problem substance. While the organizational and data exploration guide where the organization aims its ideation, the problem substance defines what the organization is searching for; that is, a set of factors that must be fully satisfied. We introduce them as part of a core assessment of the evaluation phase in section 4.2.1. Yet, these factors are also used to already guide the ideation. We further found that such ideation can be performed either in a purpose- or data-driven manner, which differ in the two explorations' order and focus. Below, we first introduce the two explorations and then integrate them into two ideation alternatives.

4.1.1. Organizational Exploration. Our interviews yielded that problem finding for ML-based AI should involve an exploration of organizational contexts to identify problems with a real and relevant organizational purpose. If such a purpose for an MLbased AI solution is missing, the experts emphasize that it becomes unlikely that the solution adds any value to the organization. While this may seem obvious, the majority of experts (63\%) also highlight that organizations often fail to question the added value of having ML-based AI solutions for their problems.

To face this issue, the experts point out that organizations can actively search for organizational purposes by pursuing two alternative trajectories: First, organizations can focus on exploring how ML-based AI may (1) replace existing solutions (79\%). In this case, organizations revisit established routines and offerings to explore whether ML-based AI may offer better ways to solve underlying problems. The experts agree that ML-based AIs can often offer valuable alternatives especially in domains in which designed solutions are bounded by humans' limited information processing capabilities or involved safety issues, or when their execution covers extensive manual efforts:

"Do I need to increase profit by selling more or do I have a lot of production costs?' Such analysis needs to be done. Whether it's manufacturing, banking, or retail, I need to know what my business is and where I can improve given solutions. Then, we can focus on how ML can help." (i9)

Second, organizations can use ML-based AI to (2) explore new problem domains (71\%) to form entirely new offerings or routines. In this case, organizations can use ML-based AI as a driver to uncover problems that were previously out of the organization's scope. While this certainly includes to explore completely new processes and offerings, the experts stress that organizations can often benefit from AI through revisiting problems that were previously unsolvable due to manual, technical, or economic limitations:

"I think you get two broad categories of either: trying to solve a problem that rendered unsolvable so far or looking for new opportunities that you didn't know existed by analyzing your environment in an open-minded way. " (i10)

4.1.2. Data Exploration. An ML-based AI's possible solution space and thus framing of potential problems is basically determined by consumable data. The experts $(88 \%)$ thus state that organizations can also guide their ideation by exploring usable data, using different foci: First, organizations can uncover potentially frameable problems along their data availability. The experts state that organizations usually start exploring data already available in electronic form. Then, they widen their focus to further sources like existing analog, public, purchasable, user, or newly recordable data (e.g., through adding novel sensors). The experts emphasize that organizations should pay special attention to high-volume data sources as this may imply a greater extent of potentially captured problem instances and thus a higher likeliness of uncovering representative data bases. Yet, the experts further stress that this allows organizations to especially uncover problem domains for which rather non-exhaustive, manually performed solutions exist due to humans' limited information processing capabilities that impede the analysis of extensive data volumes in a precise, comprehensive manner. In any case, the experts emphasize that organization must consider internal and external access restrictions (e.g., privacy issues or data ownership) to identify any access gaps as early as possible:

“'What data can we use?' Sounds trivial, but this is a huge problem. [...] Often, organizations do not even know the data they have, or it is distributed over so many systems that it would take forever to gather it. [...] What further data can we collect? What data can we additionally purchase?" (i4)

4.1.3. Purpose- \& Data-driven Ideation. While organizational and data exploration should be covered eventually, our interviews indicate that both represent alternative starting points for the ideation phase. For both approaches, organizational and data exploration represent necessary investigations to ensure clarification and inclusion of an organizational purpose and required data. However, depending on the selected approach, the experts also agree that the two exploration types are used for a different purpose; that is, either for the initial identification of potential problems or the respective subsequent exploration of a fitting organizational purpose or usable data.

The experts (92\%) state that, when following a (1) purpose-driven ideation, potential problems are 
initially derived from an organizational context. For each derived problem, potentially usable data is then explored to grasp the available technical foundation. The experts further stress that, if the required data is not available and cannot be made available in a reasonable manner, the identified potential problem should be dismissed or postponed to be solved with AI:

"The customer must have a real problem. This could be: 'We have to plan 10,000 products, but we do not have the resources to do that.' Then, this is a real problem. You can then check: 'How could we solve this with ML?' and: 'Do I have enough historic data to automate it?"' (i4)

Yet, the experts (88\%) highlight that, when following a (2) data-driven ideation instead, potential problems are first explored that build on available data. Then, organizations explore expected organizational value of its solution. If no significant purpose can be identified at this point, solving the problem should be dismissed or postponed due to its missing added value:

"We started with data. We accessed the data and investigated whether we can find things where we think that we can build something out of it. [...] If you do this without business, then this will not get you very far. You need domain knowledge to verify or falsify your hypotheses. Otherwise, it could be that you built something that works, but then they say: 'Thank you, but this does not help us at all.'” (i6)

\subsection{Evaluation Phase}

While the initial ideation phase aims to uncover potential problems, the experts $(92 \%)$ further outline a subsequent evaluation phase that aims to assess the likeliness of a problem's particular nature and context being suited to be solved with ML-based AI:

"After letting our thoughts run freely for a little bit to explore potential cases, we then narrow them down at an early stage. We try to strongly intervene to ensure that it does not go into every direction as this may result in: 'It's a nice idea, but this is not really suitable for this approach. "' (i5)

Our interviews indicate that this can be achieved with two evaluations that allow assessing the likeliness of a problem's suitability to be solved with ML-based AI. Besides, the evaluation phase also yields first indications for basic design decisions regarding $\mathrm{ML}$ based AI particularities (as we will discuss). As Figure 2 illustrates, the evaluation phase comprises two evaluations: With our interviews, we found that the evaluation phase usually starts with (1) evaluating problem substance; that is, the assessment of hard key factors that must be fulfilled to render a problem potentially solvable with ML-based AI. We further found that organizations then usually proceed with (2) evaluating problem particularities; that is, the assessment of rather soft factors that must be fulfilled in a certain degree special to a given problem to render AI solutions favorable and useful. Below, we detail the two identified assessments of the evaluation phase.
4.2.1. Evaluating Problem Substance. With our interviews, we identified key factors that the experts considered to form the substance that a problem must fulfill to be generally suited to be solved with MLbased AI. We refer to them as "substance" as they represent hard evaluation criteria that must be satisfied by each problem. Otherwise, a problem renders generally unsuited to be solved with AI. We identified five key factors that likely form the problem substance:

First, the factor that the experts (83\%) most frequently highlighted is the (a) indefinability of sufficient human-designed rules. The experts stress that if it is possible and feasible for humans to derive a sufficient solution and translate it into a set of rules, then it is usually not favorable to solve the problem using ML-based AI, but to create a solution performed manually or by a non-ML IS instead. This is, because ML-based AI solutions entail potentially detrimental properties that can render them less useful and even impractical in certain contexts (see section 4.2.2.). The experts therefore emphasize that organizations should consider ML-based AI as a second-choice problem solver that becomes only employed for problems for which their human counterparts fail to derive or articulate sufficient solutions. For instance, this is the case if humans can only define rules that cover a problem partially or must spent extensive efforts to update defined rules over time:

'First, I always ask: 'Do we really need ML?' If I can define sufficient rules, then I would always use these to better guarantee correct solution behavior. Only if such humandefined solutions are insufficient or come with high maintenance efforts, I would try to solve it with ML." (i4)

Second, a factor that the experts (71\%) also highlight is a problem's (b) self-containment in terms of its framing. The experts stress that organizations must be able to make data available that describes the aspects that are key to a problem. That is, because MLbased AIs can only observe a problem through the data that they consume and cannot consider any noncaptured aspects in their solutions. The experts further stress that a representative, self-contained problem framing does not only depend on an organization's data availability and quality, but also on the problem's nature itself. If a problem solution requires capabilities hardly capturable with statistics (e.g., intuition, long logic chains, or common sense) an ML-based AI will likely fail to find a reasonable solution within the data:

"The AI must be able to consider all information that I need to make a decision. [...] Sometimes, you want to use an AI for all steps, but you missed the multiple logical steps involved that can't be solved by a single AI." (i13)

Third, the experts $(71 \%)$ also frequently highlight that organizations must become aware that ML-based AIs only solve problems by deriving inferences through induction, i.e., deriving general, statistical 
patterns from specific observations to solve a problem. The experts thus stress that organizations must be able to frame their problems in such a way that a (c) generalization is aimed as solution. They further emphasize that organizations should deliberately evaluate whether they can expect any derivable patterns that might be integrated into a general problem solution. Otherwise, it becomes likely that their MLbased AI cannot derive a sufficient solution:

"Many projects fail because you cannot generalize related aspects well enough. It's just like that. You should carefully assess upfront whether processes, that you expect to follow a certain structure, are really likely to do it." (i6)

Fourth, another factor stated by the experts (58\%) is that organizations must be able to define a (d) clear, measurable goal of a problem to allow for an MLbased AI solution. Due to the usually large solution space that ML-based AIs explore, the experts stress the necessity of clearly defining a goal and related metrics. Otherwise, an ML-based AI will not be able to distinguish between good and bad outcomes, essentially stopping it from producing an optimized solution. The experts thus highlight that problems for which an organization's members cannot agree on the correctness of potential outcomes and how to measure the outcome's quality are not suited. Especially in subjective problem domains (e.g., rating beauty [42]), the experts stress that an agreement on what the organization perceives to be correct is inevitable:

"You must precisely define the problem: 'What exactly is the goal you're trying to achieve?' and to define an evaluation metric for that goal to grasp and agree on how a sufficient solution would look like." (i10)

Finally, a fifth key factor that the experts (54\%) emphasized is that organizations must be able to ensure a (e) continuous adaptation of an ML-based AI's solution. In particular, they stress that organizations must ensure a frequent monitoring, data collection, and retraining on more current or comprehensive data bases to let ML-based AI solutions evolve over time. Otherwise, the organizations cannot ensure wellperforming solutions if the conditions that affect related problems change or relevant exceptions are mistreated by the solutions. The experts stress that this adaptation represents a necessity for keeping the solution useful, emphasizing that organizations must evaluate whether the context allows for continuous monitoring and revision of ML-based AI solutions:

"I must be able to evaluate the results regularly to see if the solution still makes sense. Just because nobody checked if the data or business processes had changed, one of our wellworking AIs rendered completely useless over time." (i4)

4.2.2. Evaluating Problem Particularities. While the factors that comprise problem substance represent hard factors required to be fulfilled, the experts also described rather soft factors that might be acceptable in various degrees as long as a problem-specific minimum can be ensured. We refer to them as "problem particularities" as the required form of such factors is specific to a problem's particular context. Based on our interviews, we identified five factors that emerged to likely form key problem particularities. We found that such factors are usually evaluated subsequent to confirming a problem's substance.

First, as an ML-based AI solution is based on statistical generalizations, it will certainly produce errors at some point [9]. Thus, the experts (92\%) stress that the degree of (a) tolerable erroneousness for each problem context has to be evaluated. In particular, organizations should explore potential error types along with their degree of severity. The organizations should then clarify each type's maximum tolerable rate that must be ensured by a solution. The experts also emphasize that organizations must understand whether the absolute avoidance of certain errors is vital for a problem's solution to avoid a detrimental ML-based AI solution. This can also be the case if the maximum tolerable rate is not expected to be achievable or no reasonable mechanisms to intercept such errors can be identified (e.g., humans revising AIs' outputs):

"ML will always make some mistakes. So I have to ask myself: Can I allow errors to occur? How much worse is a false negative compared to a false positive and with which error rate am I willing to live? [...] Which protective layers can I build around it? " (i1)

Second, the experts (83\%) state that organizations should evaluate the relevance of (b) transparency of the inner workings and reliability of ML-based AI solutions. As the achievable transparency level varies across algorithms [13], the experts stress that organizations must understand their transparency requirements for each specific problem context. Depending on the problem, a solution must also offer the possibility to explain why a result is being provided. The experts warn that if organizations ignore any transparency requirements, their absence may create distrust among users, potentially even resulting in a complete usage refusal. Providing transparency may also be required to meet regulatory requirements:

"The lack of understanding of what is happening in the AI might be critical as algorithms may pay attention to completely different things than what we think they pay attention to. However, if and how big a problem this is actually depends on the context of its intended use." (i21)

Yet, some experts $(38 \%)$ also emphasize that organizations often ask for ungrounded, high transparency levels, even if they are not required. The experts warn that asking for excessively high transparency may restrict or even hinder achievable solutions as this can limit the design of AI solutions. 
Organizations should therefore carefully explore given problem contexts to uncover actual transparency needs:

'It's interesting that AIs' transparency is seen so critical, as we've already given up understanding many things that happen in our world - hardly anyone knows how their refrigerator works. We can evaluate AIs statistically. For many contexts this is completely sufficient to know. " (i6)

Third, a further factor that the experts (67\%) highlight is (c) fairness of AI solutions. The experts stress that organizations can use ML-based AI to actively promote more objectivity through reducing individuals' habits (e.g., prejudices or corruption) by generalizing over multiple individuals' behavior. Yet, the use of ML-based AI solutions may also create new ethical issues if AIs create discriminating behavior due to data being biased towards certain preferences and prejudices, or ethnical and social groups being badly represented in the data. The experts thus emphasize to carefully asses existent or potentially arising ethical issues within data and organizational contexts that may affect or be affected by ML-based AIs' fairness:

"Any systemically incorporated data bias will be adopted by AIs. For example, such AIs may discriminate customers that a bank's employees used to discriminate against traditionally. This needs special attention, but not everyone is aware of existing or potentially arising biases. So, we must take deliberate action to uncover discriminatory biases." (i9)

Fourth, while manual solutions or IS that are based on human-designed rules can be usually easily adapted to fulfill specific requirements, the experts $(46 \%)$ stress that solutions derived with ML-based AI may only offer a limited (d) customizability. Although it is possible to customize an AI solution by adding rules which modify its input or output, a fundamental change of the model-driven behavior can become problematic depending on the applied ML algorithm; that is, because conducting adaptations of more complex, nontransparent algorithms (e.g., neural networks) requires indirect changes through retraining AIs with a changed goal or data and thus knowledge of data-science techniques in combination with domain and data understanding. The experts therefore emphasize that organizations must carefully assess whether problems have to meet any potential requirements that might be too specific to be ensured at the core of an AI solution:

"Our AI recognized a billion documents correctly, but then we had one type that didn't want to work right. It can be an incredible effort to also correctly get this type while ensuring the correctness of the other cases. In traditional programming, I can just add a rule to handle this. But if I treat exceptions in my AI, I can't just handle that exception, I actually start to solve the problem all over again." (i17)

Lastly, a fifth factor stated by the experts $(42 \%)$ is the achievable (e) response time of an AI solution. Depending on the data volume that has to be processed and ML algorithms' processing time, the experts stress that a solution's response time may vary widely. As with human solutions however, organizations must ensure to provide a response as soon as it is required in the specific context. A gradual solution might not only render produced outcomes useless but may even cause fatal consequences (e.g., delayed warnings). Thus, organizations should carefully evaluate required minimum response times to clarify whether available data and algorithms likely allow for a fitting solution:

"If an organization wants to get a result every five minutes, then, of course, it has to be ready within five minutes. That's something you must always actively examine: 'How often do you need a result? What is the time horizon? Is it even realistic that we do the inference in time?' Depending on the ML algorithm you want to use, the inference can take a while." (i18)

\section{Discussion}

With our study, we explored how problem finding translates to organizational contexts in which solutions are not purely derived by humans, but by ML-based AIs. Through interviewing 24 experts that regularly conduct AI initiatives, we found first evidence for essential procedural artifacts and related key factors. We synthesized our findings to propose a basic problem-finding framework that is contextualized for ML-based AI problem solving.

We can offer several theoretical contributions. To the best of our knowledge, we are among the first to study how the essential problem finding translates to organizational ML-based AI solver contexts. By providing initial findings on how to identify problems and evaluate their suitability to be solved with MLbased AI, we answer recent calls for research on how to manage the emerging human-machine symbioses in cognitive organizational contexts [e.g., 11, 40, 44, 50]. Moreover, our findings qualify the recently emerging lines of IS research that examine ML-based AI particularities, such as research on fair [e.g., 1, 30, 42] and explainable AI [e.g., 13, 31, 38], and confirm the relevance of their consideration even at the earliest project stage of problem finding. Our findings further confirm that the fundamental ideation and evaluation phases of purely human-driven problem finding also form key phases in AI solver contexts. Lastly, as we identified key characteristics of AI-suited problems, we hope to inform future research on innovation, design, and diffusion of ML-based AI in organizations.

Our study also comprises significant contributions for practitioners. Organizations can use our findings to better manage their problem-solving activities when their humans and machines jointly contribute to problem solving. In particular, organizations can apply the proposed framework to explore and evaluate problems underlying potential AI initiatives in a structured manner. Our findings can help organizations 
to better uncover possibilities to exploit ML-based AIs' potential for enhancing processes and offerings. Organizations can also use the framework to protect themselves already at an early stage from mistakenly promoting AI-driven problem-solving initiatives that are not suited for deploying ML-based AI.

Of course, our study is subject to some limitations. First, we did not perform empirical testing of the proposed framework. Here, future studies should focus on evaluating both the procedural artifacts and identified key factors. As we chose to pursue a rather general perspective, future studies can test our findings' applicability in contexts with special requirements to further contextualize the model (e.g., highly serious or subjective contexts, such as medical or recruiting solutions). Second, while we tried to ensure a wide-ranging analysis, the resulting set of proposed factors is rather a non-exhaustive list. As we focused on exploring key artifacts and factors, further analyses that offer additional explorable contexts and problem characteristics represent fruitful avenues for future research. Third, although our interviewees cover a wide range of roles and industries, we cannot completely rule out any data biases. To reveal such biases, quantitative studies in varying contexts can be used to further validate our framework's applicability.

Presently, we do not know much about how MLbased AI will change organizational problem solving. Yet, history showed us that ML-based AI is able to build brilliant solutions if applied to the right problems. Furthering our understanding of how to effectively uncover suitable problems may therefore play a crucial role in ultimately unlocking the full potential of AI.

Acknowledgements. This study has been funded by the German Federal and Hessen State Ministry for Education and Research within their support of the National Research Center for Applied Cybersecurity ATHENE.

\section{References}

[1] Afrashteh, S., M. Davern, and I.A. Someh, "Enhancing Fairness in Algorithmic Decision-Making Through Perspective Taking”, ECIS Proceedings, (2020).

[2] Agrawal, A., J.S. Gans, and A. Goldfarb, "What to Expect From AI”, MIT Sloan Mgmt. Review 58(3), 2017.

[3] Amershi, S., A. Begel, C. Bird, et al., "Software engineering for machine learning: A case study", IEEE/ACM ICSE Proceedings, (2019), 291-300.

[4] Basadur, M.S., G.B. Graen, and S.G. Green, "Training in creative problem solving in an industrial research org.", Organ. Behav. Hum. Perform. 30, 1982, pp. 41-70.

[5] Bean, R., "Demystifying AI in the Corporation", 2019. https:/www.forbes.com/sites/ciocentral/2019/10/09/demystif ying-artificial-intelligence-ai-in-the-corporationforbes/\#7123b2606016 (acc. Nov. 16, 2020).
[6] Benta, C., J. Wilberg, C. Hollauer, and M. Omer, "Process model for data-driven business model generation", ICED Proceedings, (2017), 347-356.

[7] Björk, J., "Knowledge domain spanners in ideation", Creativity and Innovation Mgmt. 21(1), 2012, pp. 17-27.

[8] Bremser, C., "Starting points for big data adoption", ECIS Proceedings, (2018).

[9] Brynjolfsson, E., and T. Mitchell, "What can machine learning do? Workforce implications", Science 358(6370), 2017, pp. 1530-1534.

[10] Chi, M., P. Feltovich, and R. Glaser, "Categorization and representation of physics problems by experts and novices", Cognitive Science 5(2), 1981, pp. 121-152.

[11] Coombs, C., D. Hislop, S.K. Taneva, and S. Barnard, "The strategic impacts of Intelligent Automation for knowledge and service work: An interdisciplinary review", Journal of Strategic Information Systems 101600, 2020.

[12] Delbecq, A., and A. Van de Ven, "A Group Process Model for Problem Identification and Program Planning", Journal of Applied Behavioral Sci. 7(4), 1971, pp. 466-492.

[13] Diakopoulos, N., “Accountability in algorithmic decision making”, Comm. ACM 59(2), 2016, pp. 56-62.

[14] Domingos, P., "A Few Useful Things to Know About Machine Learning", Comm. ACM 55(10), 2012, pp. 78-87.

[15] Van den Ende, J., L. Frederiksen, and A. Prencipe, "The front end of innovation: Organizing search for ideas", Journal of Product Innov. Mgmt. 32(4), 2015, pp. 482-487.

[16] Fedyk, A., "How to tell if machine learning can solve your business problem", 2016. https://hbr.org/2016/11/howto-tell-if-machine-learning-can-solve-your-business-problem (acc. Nov. 16, 2020).

[17] Flick, U., "Triangulation in qualitative research", In $A$ companion to qualitative research. Sage Publications., London, UK, 2004, 173-183.

[18] Forbes Insights, "On Your Marks: Business Leaders Prepare For Arms Race In Artificial Intelligence”, 2018. https://www.forbes.com/sites/insights-intelai/2018/07/17/onyour-marks-business-leaders-prepare-for-arms-race-inartificial-intelligence/\#4084b00f1946 (acc. Nov. 16, 2020).

[19] He, K., X. Zhang, S. Ren, and J. Sun, “Delving deep into rectifiers: Surpassing human-level performance on imagenet classification", IEEE ICCV Proceedings, (2015), $1026-1034$.

[20] Hippel, E. von, and G. von Krogh, "Problem solving without problem formulation", Organization Science 27(1), 2016, pp. 207-221.

[21] Hsieh, H.F., and S.E. Shannon, "Three approaches to qualitative content analysis", Qualitative Health Research 15(9), 2005, pp. 1277-1288.

[22] Jordan, M.I., and T.M. Mitchell, "Machine learning: Trends, perspectives, and prospects", Science 349(6245), 2015, pp. 255-260.

[23] Kayser, V., B. Nehrke, and D. Zubovic, "Data Science as an Innovation Challenge: From Big Data to Value Proposition", Techn. Inn. Mgmt. Rev. 8(3), 2018, pp. 16-25. 
[24] Kolb, D.A., "Management and the Learning Process", California Management Review 18(3), 1976, pp. 21-31.

[25] Kruse, L., N. Wunderlich, and R. Beck, "AI for the Financial Services Industry: What Challenges Organizations to Succeed", HICSS Proceedings, (2019).

[26] Kurgan, L.A., and P. Musilek, "A survey of knowledge discovery and data mining process models", Knowledge Engineering Review 21(1), 2006, pp. 1-24.

[27] Lang, J.R., J.E. Dittrich, and S.E. White, "Managerial Problem Solving Models: A Review and a Proposal", Academy of Management Review 3(4), 1978, pp. 854-866.

[28] Leavitt, H.J., "Beyond the Analytic Manager", California Management Review 17(3), 1975, pp. 5-12.

[29] Lyles, M., and I.I. Mitroff, “Org. Problem Formulation: An Empirical Study”, Adm. Sci. Q. 25(1), 1980, pp. 102.

[30] Martin, K., "Designing ethical algorithms", MIS Quarterly Executive 18(2), 2019, pp. 129-142.

[31] Miller, T., "Explanation in AI: Insights from the social sciences", Artificial Intelligence 267, 2019, pp. 1-38.

[32] Mitchell, T.M., Machine Learning, McGraw-Hill, New York, NY, 1997.

[33] Mumford, E., and 1998, "Problems, knowledge, solutions: solving complex problems", The Journal of Strategic Information 7(4), 1998, pp. 255-269.

[34] Mumford, M., R. Reiter-Palmon, and M. Redmond, "Problem construction and cognition: Applying problem representations in ill-defined domains.", In Creativity research. Problem finding, problem solving, and creativity. Ablex Publishing, 1994, 3-39.

[35] Mumford, M.D., D.L. Whetzel, and R. Reiter-Palmon, "Thinking creatively at work: Org. influences on creative problem solving", J. Creat. Behav. 31(1), 1997, pp. 7-17.

[36] Myers, M.D., and M. Newman, "The qualitative interview in IS research: Examining the craft", Information and organization 17(1), 2007, pp. 2-26.

[37] Osborn, A.F., Applied imagination, Charles Scribner's Sons, New York, 1953.

[38] Peters, F., L. Pumplun, and P. Buxmann, "Opening the Black Box: Consumer's Willingness to Pay for Transparency of Intelligent Systems", ECIS Proceedings, (2020).

[39] Pumplun, L., C. Tauchert, and M. Heidt, “A New Organizational Chassis for AI - Exploring Organizational Readiness Factors”, ECIS Proceedings, (2019).

[40] Rai, A., P. Constantinides, and S. Sarker, "Editor's Comments: Next-Generation Digital Platforms: Toward Human-AI Hybrids", MIS Quarterly 43(1), 2019, pp. iii-ix.

[41] Reiter-Palmon, R., and E.J. Robinson, "Problem Identification and Construction: What Do We Know, What Is the Future?", Psychology of Aesthetics, Creativity, and the Arts 3(1), 2009, pp. 43-47.

[42] Rhue, L., "Beauty is in the AI of the Beholder: How AI Anchors Human Decisions on Subjective vs. Objective Measures", ICIS Proceedings, (2019).

[43] Russell, S.J., and P. Norvig, Artificial Intelligence: A
Modern Approach, Addison Wesley, Boston, MA, 2016.

[44] Rzepka, C., and B. Berger, "User Interaction with AIenabled Systems: A Systematic Review of IS Research", ICIS Proceedings, (2018).

[45] Saldaña, J., The Coding Manual for Qualitative Researchers, Sage Publications, London, UK, 2015.

[46] Samuel, A.L., "Some studies in machine learning using the game of checkers", IBM Journal of Research and Development 3(3), 1959, pp. 210-229.

[47] Sarker, S., X. Xiao, and T. Beaulieu, "Qualitative studies in information systems: a critical review and some guiding principles", MIS Quarterly 37(4), 2013, pp. iii-xviii.

[48] Satell, G., "How to Make an AI Project More Likely to Succeed.", 2018. https://hbr.org/2018/07/how-to-make-an-aiproject-more-likely-to-succeed (acc. Nov. 16, 2020).

[49] Schmelzer, R., "The AI-enabled Future”, 2019. https://www.forbes.com/sites/cognitiveworld/2019/10/17/theai-enabled-future/\#1260a9a53339 (acc. Nov. 16, 2020).

[50] Schuetz, S., and V. Venkatesh, "Research perspectives: The rise of human machines: How cognitive computing systems challenge assumptions of user-system interaction", $J$. Assoc. Inf. Syst. 21(2), 2020, pp. 460-482.

[51] Silver, D., J. Schrittwieser, K. Simonyan, et al., "Mastering the game of Go without human knowledge", Nature 550(7676), 2017, pp. 354-359.

[52] Simon, H.A., "The structure of ill structured problems, Artificial Intelligence", Artificial Intelligence 4(3-4), 1973, pp. 181-201.

[53] Simon, H.A., and A. Newell, "Human Problem Solving: The State of the Theory in 1970", American Psychologist 26(2), 1971, pp. 145-159.

[54] Smith, G.F., "Defining Managerial Problems: A Framework for Prescriptive Theorizing", Management Science 35(8), 1989, pp. 963-981.

[55] Sturm, T., and F. Peters, "The Impact of AI on Individual Performance: Exploring the Fit between Task, Data, and Technology", ECIS Proceedings, (2020).

[56] Taylor, R.N., "Perception of Problem Constraints", Management Science 22(1), 1975, pp. 22-29.

[57] Traumer, F., S. Oeste-Reiß, and J. Leimeister, "Towards a Future Reallocation of Work between Humans and Machines - Taxonomy of Tasks and Interaction Types in the Context of Machine Learning", ICIS Proceedings, (2017).

[58] Vanauer, M., C. Bohle, and B. Hellingrath, "Guiding the introduction of big data in organizations: A methodology with business- and data-driven ideation and enterprise architecture management-based implementation", HICSS Proceedings, (2015), 908-917.

[59] Vinyals, O., I. Babuschkin, W. Czarnecki, et al., "Grandmaster level in StarCraft II using multi-agent reinforcement learning”, Nature 575(7782), 2019, pp. 350 354.

[60] Weber, R.P., Basic content analysis, Sage Publications, London, UK, 1990. 